\title{
Metastability of Mechanically Alloyed and Spark Plasma Sintered $\mathrm{Co}_{50} \mathrm{Ti}_{50}$ Powder
}

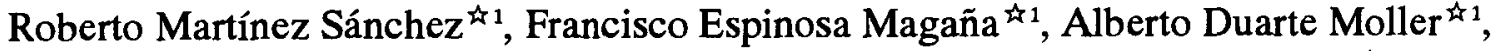 \\ José Gerardo Cabañas Moreno ${ }_{\text {ฟ2 }}$, David Rios Jara ${ }^{\text {मै } 1}$, Yoshito Nishikawa ${ }^{\text {मै }}$ \\ and Sebastián Díaz De la Torre ${ }^{41,3}$. \\ ฬ1 1 Advanced Materials Research Center CIMAV. Miguel de Cervantes 120 CP.31110. Chihuahua Mexico. \\ 22 National Polytechnique Institute. ESFM-UPALM. CP.07738, Mexico, D.F., Mexico. \\ 43 ${ }^{3}$ Technology Research Institute of Osaka Prefecture. 2-7-1 Ayumino, Izumi city, 594-1157 Osaka Japan.
}

Received January 24,2000

\section{SYNOPSIS}

High purity cobalt and titanium powders were equiatomically mixed and mechanically alloyed. The microstructure and phase transformation occurred on the powder product during its densification using the spark plasma sintering SPS technique were analyzed. CoTi and $\mathrm{CoTi}_{2}$ intermetallic compounds formed after fast sintering. Although metastable $\mathrm{CoTi}_{2}$ undergoes partial CoTi transformation the lattice parameter and micro-Vickers hardness of these sintered compounds show high stability when annealing from 973 to $1273 \mathrm{~K}$. The material thus prepared can be reliably used at these temperatures showing excellent mechanical behavior.

KEYWORDS

Mechanical Alloying, Plasma, Sintering, Co, Ti, Intermetallic

\section{Introduction}

The mechanical alloying (MA) process is an effective method to develop strategic materials with novel structures. These can be solid solutions (even in immiscible systems), amorphous phases, fine particle sized and nano-metric crystals, etc. ${ }^{1-5)}$. The MA products are usually processed by conventional powder metallurgy routes before being consolidated. The novel features conferred into the powders, however, such as fine crystal size and chemical homogeneity can be lost during sintering operations, due to the high thermodynamic activity of the synthesized phases. Indeed, grain growth can hardly be prevented during the sintering process unless rapid densification is performed. The spark plasma sintering (SPS) process is an alternative route to dense mechanically alloyed products at a high grade while retaining their novel structures.

Advantages of the SPS process over other conventional sintering processes are the fast heating and cooling rates attained, and high pressure applied during the sintering course. This makes possible to obtain highly densified products in short times ${ }^{6,7}$. Combining these two material processing techniques (MA and SPS) it is possible to produce materials with unique microstructures for industrial applications. Previous studies on Co-Ti alloys ${ }^{3-5,8)}$ processed by MA and SPS have shown that the final products are composed of mixtures of metastable intermetallic compounds mostly developed during crystallization of the amorphous phase produced. Co-Ti intermetallic compounds offer a combination of high melting point with low to medium density and high oxidation resistance?). In addition, the CoTi phase is one of the few B2 (ClCs structure) intermetallic compounds that show a positive temperature dependence with respect to the yield strength, i.e., between 400 and $1073 \mathrm{~K}^{9}$. These features make it potential candidates for medium-elevated temperature applications.

Furthermore, Co-Ti intermetallic compounds show some degree of ductility at room temperature making them even more interesting from a microstructural point of view. Previously, it was found out that up to 1.0 at.\% of Fe could be released from the milling media and incorporated into the lattice structure of the Co-Ti precursor powder when milling the mixture for $4 h^{3}$. To some extent, such Fe content could distort the final lattice parameter of the intermetallic compounds formed, but did not play an important role upon the powder amorphizationcrystallization sequence ${ }^{3)}$. In this work, the equiatomic $\mathrm{Co}_{50} \mathrm{Ti}_{50}$ alloy was mechanically alloyed for longer times and microstructurally characterized after spark plasma sintering and annealing at different temperatures to study metastability (phase transformation) of bulk bodies.

\section{Experimental procedures}

Co-Ti alloys were produced by mechanical alloying the 
powder mixtures with nominal composition $\mathrm{Co}_{50} \mathrm{Ti}_{50}$ (at.\%) in an attrition milling device. The ball-to-powder weight ratio was set to $65: 1$. All experiments were performed at $350 \mathrm{rpm}$. Argon gas flow was maintained inside the milling vial during experimental runs. The milling time was set to $20 \mathrm{~h}$ in all cases. Details on the characterization of the asmilled powders can be found in related publications ${ }^{45}$.

Densification of the powder products was conducted using a Dr. Sinter SPS equipment. Five grams of as-milled powders were placed in a cylindrical graphite die. The sintering conditions were: heating and cooling rates of 1.5 $\mathrm{K} / \mathrm{sec}$. The sample was held at $1173 \mathrm{~K}$ and $50 \mathrm{MPa}$ for 5 min and cooled down immediately. Annealing treatments were carried out for $1 \mathrm{~h}$ at $973,1073,1173$ and $1273 \mathrm{~K}$ under argon atmosphere, with heating and cooling rates of $0.166 \mathrm{~K} / \mathrm{sec}$. Samples were characterized in the as-sintered and annealed conditions by means of $\mathrm{X}$-ray diffraction (XRD) using a Siemens D-5000 diffractometer and $\mathrm{Cu}_{-\mathrm{K} \alpha}$ rad. $(\lambda=0.15405 \mathrm{~nm})$. Scanning electron microscopy (SEM) was performed using a JEOL JEM-5800 LV equipment, supplied with a energy-dispersive spectrometer (EDS). Hardness tests were conducted using a $200 \mathrm{~g}$ loadindenter during $25 \mathrm{sec}$ with a FM Future-Tech Japan microhardness tester.

\section{Results and discussion}

After milling the powder mixture for $20 \mathrm{~h}$ XRD analysis revealed the formation of an amorphous-like pattern which included Co and Ti peaks. These peaks were substantially broad and short suggesting an amorphous matrix having nano-scaled $\mathrm{Co}$ and Ti particles embedded ${ }^{3}$. The presence of about 3.5 at.\% Fe in the sintered samples of this study was revealed through EDS microanalysis. Moreover, a decrease in the Co content was observed, which is probably due to its alloying with the container walls and balls during attrition, and also probably due to mechanical drag, as reported before ${ }^{4,8)}$. Thus, the original $(1: 1) \mathrm{Co}$ : Ti ratio changed. Table 1 shows the chemical analysis of the original and as-sintered compositions, including the standard deviation determined by EDS. The analysis was carried out over areas of about $100 \times 100 \mu \mathrm{m}^{2}$.

The microstructure of the consolidated sample (before

Table 1 Elemental composition (at.\%) of original and sintered specimens (* determined by EDS).

\begin{tabular}{|c|c|c|}
\hline Element & Original & As-sintered $*$ \\
\hline $\mathrm{Co}$ & 50.0 & $42.1 \pm 0.15$ \\
\hline $\mathrm{Ti}$ & 50.0 & $54.4 \pm 0.30$ \\
\hline $\mathrm{Fe}$ & 0.0 & $3.5 \pm 0.20$ \\
\hline
\end{tabular}

annealing) is shown in Fig. 1. In this figure two main regions were observed. EDS microanalysis conducted in these zones indicated that the one in major extent corresponds to the bright phase ( $\left(1^{\text {st }}\right.$ zone), whose composition is rich in $\mathrm{Ti}$ (Table 2). The main features of this phase are the low Fe content and a Co: Ti ratio close to the originally set ratio. A dark second region ( $2^{\text {nd }}$ zone) detected in Fig. 1 was found surrounding the bright regions. It disclosed larger $\mathrm{Fe}$ content and also smaller grain sizes than the bright phase. It has a composition that is closer to the one resulted in the as-sintered sample, whereas its $\mathrm{Co}$ : Ti ratio is far from to the original ratio (Table 2).

Further SEM analysis revealed the presence of a third phase ( $3^{\text {rd }}$ zone). This was found inside of some zones of the bright phase described above (see Fig.2). An important characteristic of this phase is that it has the highest level of Ti whereas the $\mathrm{Fe}$ content estimated is lower than 0.4 at.\%.

Fig. 3 shows a collection of X-ray diffraction (XRD) patterns corresponding to the as-sintered specimen and those recorded after annealing such specimens at different temperatures. The XRD pattern of the specimen in the assintered condition shows mixture of at least two phases. The phases that were identified correspond to the intermetallic compounds CoTi and $\mathrm{CoTi}_{2}$. Shortening of

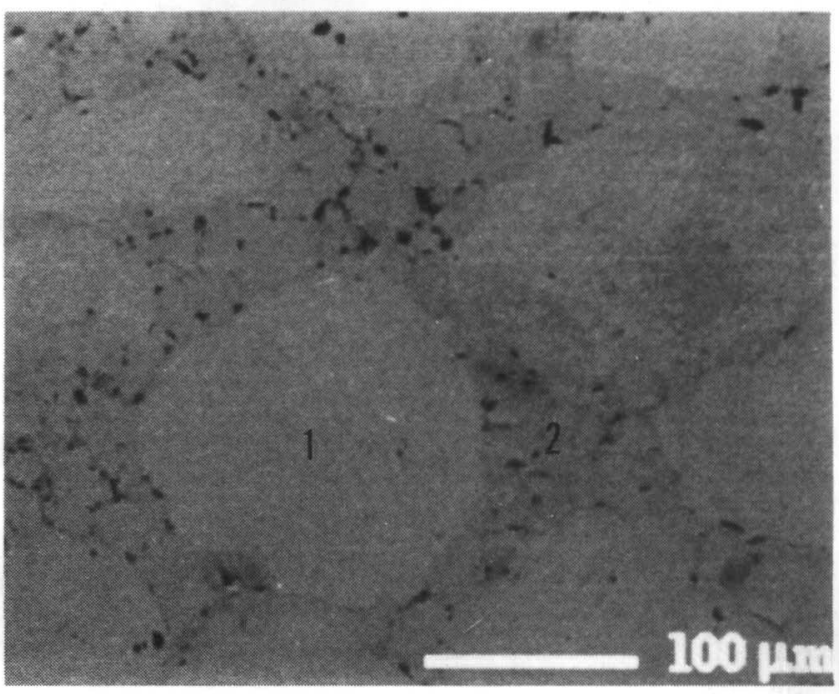

Fig.1 SEM micrograph of the as-sintered specimen showing two main different zones analyzed (Table 2).

Table 2 Microanalysis in marked zones of Figs. 1 and 2, in at.\% (as-sintered specimens).

\begin{tabular}{|c|c|c|c|c|}
\hline Zone & $\mathrm{Co}$ & $\mathrm{Ti}$ & $\mathrm{Fe}$ & $\begin{array}{c}\text { Co:Ti } \\
\text { ratio }\end{array}$ \\
\hline 1- bright & 47.72 & 51.92 & 0.36 & $0.90: 1$ \\
\hline 2- dark & 40.56 & 54.04 & 3.40 & $0.75: 1$ \\
\hline 3- dark & 32.76 & 66.88 & 0.39 & - \\
\hline
\end{tabular}


the diffraction peaks is evident after annealing. Note that the $\mathrm{CoTi}_{2}$-peak intensities of the specimen heated at the highest temperature nearly vanished, which suggests both partial transformation and grain size relaxation of the $\mathrm{CoTi}_{2}$ phase. Evidently the microstructure of sintered bodies was kept in a non-stable state.

Annealing temperature enhanced Ti-atoms diffusion from zone 1 to 2 and ultimately 3 . Simultaneously, Co atoms need to be removed in the opposite direction, i.e., from zone 3-2-1. That is why the Co:Ti ration modified. Estimation of the mass fraction of these two intermetallics in the as-sintered sample using Rietveld analysis gave values of 90.9 at.\% CoTi and 9.1 at.\% $\mathrm{CoTi}_{2}$. Similar mass fraction evaluations were carried out on the annealed

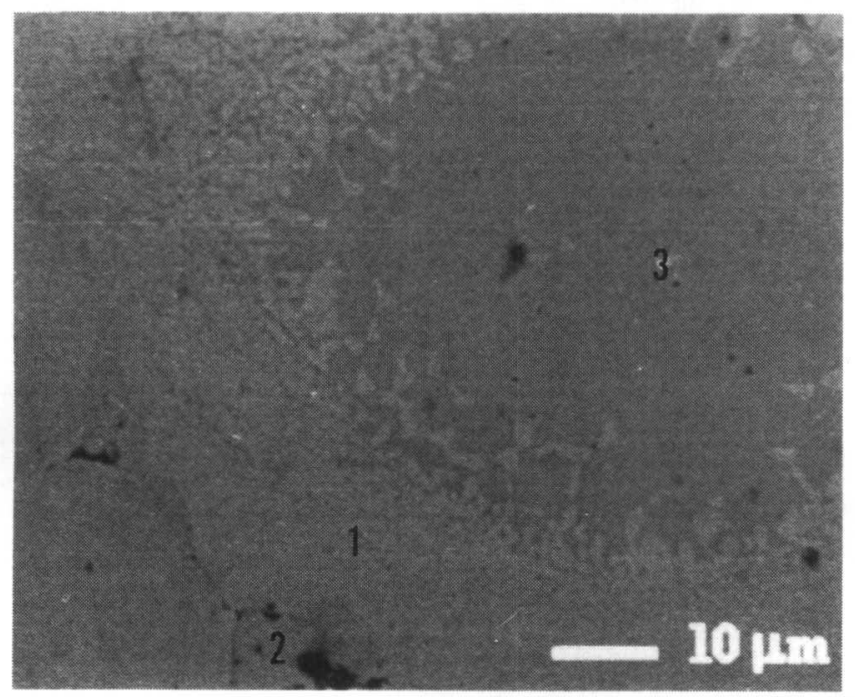

Fig.2 SEM micrograph of the as-sintered specimen showing a third dark phase precipitated inside the bright phase of Fig. 1.

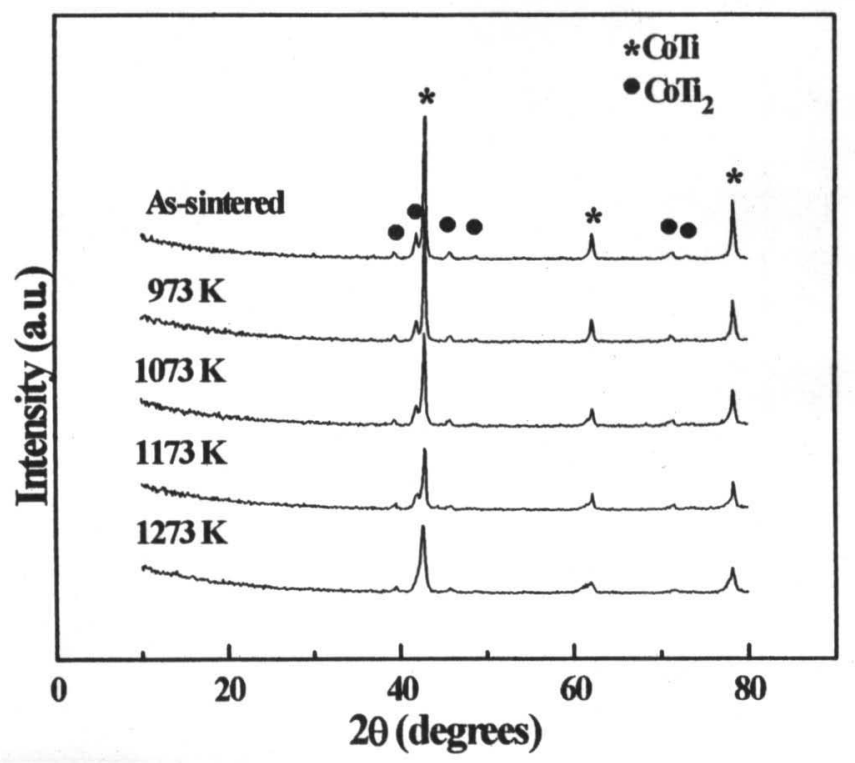

Fig.3 XRD-patterns of as-sintered $\mathrm{Co}_{50} \mathrm{Ti}_{50}$ before and after annealing. samples. For the sample annealed at $1273 \mathrm{~K}$ the results indicated 96.8 at.\% and only 3.2 at.\% for CoTi and $\mathrm{CoTi}_{2}$, respectively. After annealing, the CoTi intermetallic compound was found the predominant and more stable phase. This phenomena implies that: a) the $\mathrm{CoTi}_{2}$ decomposes to form more CoTi, b) the initial as-sintered composition is close to the stoichiometry of $\mathrm{Co}_{50} \mathrm{Ti}_{50}, \mathrm{c}$ ) the CoTi intermetallic compound has a more simple structure than $\mathrm{CoTi}_{2}$, (Table 3 shows the main differences found between these structures), and d) at this composition the thermodynamical stability of CoTi is higher than $\mathrm{CoTi}_{2}$. On the other hand, previous thermodynamical studies in the Co-Ti system ${ }^{12)}$ had shown, using the Miedema model, that the equiatomic mixture exhibits more stability than other mixtures with different $\mathrm{Co}$ : Ti ratio, independently of temperature and at the chosen composition (Table 1) of this work.

The stabilization of CoTi intermetallic compound after annealing confirms previous thermodynamic results ${ }^{3}$. The intermetallic compounds crystallized during the sintering process showing a short-range diffusion order. The $\mathrm{CoTi}_{2}$ complex structure (cF96) is eager to adopt a more simple atomic arrangement, i.e., the cP2, Pm3m symmetry having 2 atoms/unit cell.

The variation of the lattice parameter of CoTi and $\mathrm{CoTi}_{2}$ compounds was measured on its initial condition and after annealing. The results are reported in Table 4 . The lattice parameters of the as-sintered sample indicated $0.296 \mathrm{~nm}$ for the CoTi compound and $1.122 \mathrm{~nm}$ for $\mathrm{CoTi}_{2}$. Both cases being slightly different from those reported in JCPDS ${ }^{13}$ ) standards.

Table 3 Differences between the studied intermetallic compounds.

\begin{tabular}{|c|c|c|c|}
\hline Phase & $\begin{array}{c}\text { Pearson's } \\
\text { symbol }\end{array}$ & Atoms/cell & Symmetry \\
\hline $\mathrm{CoTi}$ & $\mathrm{cP} 2$ & 2 & $\mathrm{Pm} 3 \mathrm{~m}$ \\
\hline $\mathrm{CoTi}_{2}$ & $\mathrm{cF} 96$ & 96 & $\mathrm{Fm} 3 \mathrm{~m}$ \\
\hline
\end{tabular}

Table 4 Variation in lattice parameter and microhardness of specimens after annealing.

\begin{tabular}{|c|c|c|c|}
\hline & \multicolumn{2}{|c|}{$\begin{array}{c}\text { Lattice parameter } \\
(\mathrm{nm})\end{array}$} & $\begin{array}{c}\text { Micro-hardness } \\
(\mathrm{Hv})\end{array}$ \\
\hline Condition & $\mathrm{CoTi}$ & $\mathrm{CoTi}_{2}$ & - \\
\hline JCPDS ref. & 0.298 & 1.130 & - \\
\hline As-sintered & 0.296 & 1.122 & $632.6 \pm 93.2$ \\
\hline $973 \mathrm{~K}$ & 0.299 & 1.120 & $649.4 \pm 72.4$ \\
\hline $1073 \mathrm{~K}$ & 0.299 & 1.120 & $650.0 \pm 71.6$ \\
\hline $1173 \mathrm{~K}$ & 0.299 & 1.120 & $654.7 \pm 83.1$ \\
\hline $1273 \mathrm{~K}$ & 0.299 & - & $651.6 \pm 93.1$ \\
\hline
\end{tabular}


The lattice parameter values of those intermetallics, followed as a function of the annealing temperature can also be deduced from Table 4 .

Fig. 4 shows a SEM micrograph of a sintered sample annealed at $1273 \mathrm{~K} / 1 \mathrm{~h}$. The specimen could be nearly homogenized after the heat treatment since the mixture of phases was not even confirmed by XRD. In practice, the best homogenization was attained for the sample heated to $1273 \mathrm{~K}$. Considering that for this specimen, the X-ray peaks diffracted from the $\mathrm{CoTi}_{2}$ intermetallic compound almost disappeared, and also that during the lattice parameter estimations was not possible to accurately analyze the same reflections as in the as-sintered case, we assume the $\mathrm{CoTi}_{2}$ lattice parameter value to be somehow less reliable than the values determined for other specimens. Although the lattice parameter data calculated in this work for both intermetallics reveal very small variations after the annealing operations, the differences between these values might be neglected. This lead us to note that an apparent stabilization occurred during annealing at low temperatures. Although there are no current reports specifying the whole Co-Ti-Fe equilibrium ternary diagram, it might be possible that the CoTi intermetallic compound can have dissolved more quantity of $\mathrm{Fe}$.

Table 4 summarizes hardness $(\mathrm{Hv})$ data of the sintered specimens as a function of the annealing temperature. Statistical hardness-analysis was made with $95 \%$ confidence. All samples show high Hv values. Although nearly negligible, the observed $\mathrm{Hv}$ variation is mainly attributed to the degree of densification attained, however, it must also be closely influenced by the kind of phases present and their concentration. Note that in all specimens more than 90 at.\% corresponds to CoTi. Thus, the resulting high hardness values may also be attributed to the ample presence of this compound.

\section{Conclusions}

Sintered products consist of a mixture of two intermetallic compounds; namely $\mathrm{CoTi}_{2}$ and CoTi. Although the nominal composition has an important effect on the phases transformation sequence during annealing, the most stable phase (CoTi) predominates. It was not observed any detrimental effect caused by the presence of 3 at.\% $\mathrm{Fe}$ in the transformation sequence. Apparently, $\mathrm{Fe}$ slightly alters the lattice parameter. After annealing the sintered bodies the variation in both hardness and lattice parameters was small. Annealing improved microstructural homogenization and stability of the bulks.

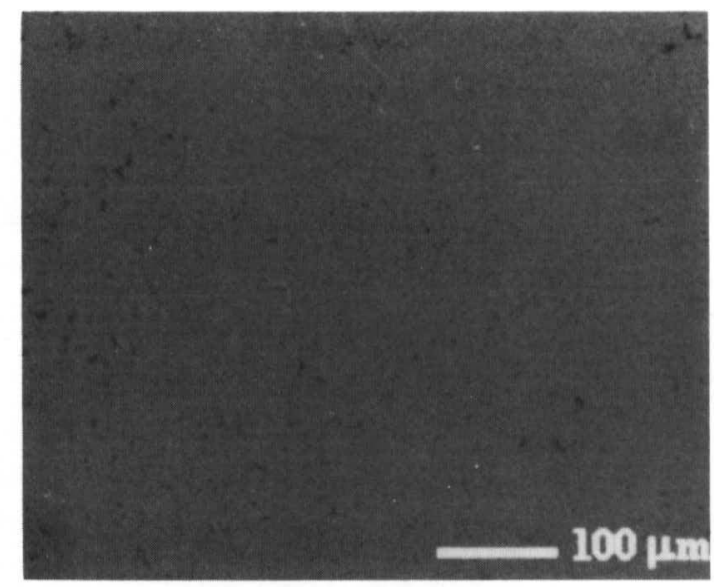

Fig.4 Typical SEM microstructure of the as-sintered specimen after annealing at $1273 \mathrm{~K}$.

\section{References}

1) C.C.Koch: Annual Review of Mat. Sci. and Eng.-A, 13(1983)121-143.

2) C.C.Koch: Mat. Sci. and Eng.-A., 244(1998)39-48.

3) R.Martínez S., J.Cabañas-M., D.Rios-J. and S.D.De la Torre: J. Japan Soc. of Powder Metallurgy, 46(1999) 942-946.

4) J.Cabañas-M., R.Martínez-S., H.A.Calderón, M. Umemoto and S.Shiga: Proc. Processing. and Properties of Nanocrystalline Materials, USA. (Suryanarayana, J.Singh and F.H.Froes Eds.), (1996)319-329.

5) J.Cabañas-M., R.Martínez-S., H.A.Calderón, H.Balmori-R. and M.Umemoto: Mat. Sci. Forum, 225227(1996)435-440.

6) N.Tamary, T.Tanaka, K.Tanaka, I.Kondoh, M.Kawahara and M.Tokita: Journal of the Ceramic Society of Japan, 103(1995)740-742.

7) M.Tokita: J. Japan Soc. of Powder Metallurgy, 30 (1996)790-804.

8) R.Martínez-S., J.Cabañas-M, H.A.Calderón, H. Mendoza, Bokhimi, M.Umemoto, S.Shiga and V.M. López-H.,: Mat. Sci. and Eng.-A., 228(1997)37-40.

9) T.Takasugi and O.Izumi: Acta Metall., 33(1985)39-48.

10) S.D.de la Torre, D.Olezak, A.Kakitsuji, K.Miyamoto, H.Miyamoto, R.Martínez-S, F.Almeraya-C., A.Martínez-V. and D.Ríos-J.: Mat. Sci. and Eng.-A., 276(2000)232-241.

11) J.L.Murray: "Phase Diagrams of Binary Titanium Alloys", Ed T.B.Massalky, (Metals Park, Oh, ASM International), (1987)59-68.

12) Y.Liu, T.Takasugi and O.Izumi: Metallurgical Trans., 17-A(1986)1433-1439.

13) JCPDS, cards $18-429$ and 7-141. 